\title{
An Improved Ray Casting Algorithm Based on Morphological Segmentation of Multiple Structure Elements
}

\author{
Huiling Hou ${ }^{*}$, Mingquan Wang, Xiaopeng Wang and Jinkun Lv
}

National Key Laboratory for Electronic Measurement Technology, North University of China, Taiyuan, 030051, China

\begin{abstract}
Aim at traditional ray casting algorithm existing the problems that powerful computing capacity and slow rendering velocity, an improved ray casting algorithm is presented in this paper. To reduce the number of voxels in reconstruction process, the image segmentation is introduced to volume rendering. A segmentation algorithm based on multiple structure elements is proposed to process ICT slice images original data field. The original voxels are divided into two parts: the target voxel data set and the background voxel data set. Then, the target voxel data set is constructed as a new data field, and using the ray casting algorithm to it. This reduces the number of voxels and accelerate the efficiency of ray casting. The results of reconstruction show that this algorithm can greatly reduce reconstruction volume data and improve the efficiency of ray casting.
\end{abstract}

Keywords: ICT, multiple morphological structure elements, ray casting algorithm.

\section{INTRODUCTION}

Visualization in Scientific Computing (ViSC) is a new important research direction of computer graphics. The main idea is converting a large number of engineering test data which generated by engineering operations into $2 \mathrm{D}$ graphics or $3 \mathrm{D}$ images by using the basic principles of computer graphics $[1,2]$. Visualization technology in ICT converts 2D CT test data into 3D stereo images, which intuitively reveal workpiece defects. It makes engineering staffs have multiangle and multi-level observation. Also, it can enhance the ability of detection and provide more comprehensive and precise analysis results to non-destructive testing [3]. Therefore, visualization technology in ICT has important practical significance and research value. javascript:void(0);

The ray casting algorithm is the classic algorithm of volume rendering technology, which has been widely researched and applied. The simple principle and the easy realization are the main advantage of the ray casting algorithm. It can also keep the details of 3D image, enhance the overall rendering effect and generate high quality images to display. But visualization speed still can not satisfy with constantly improving in industrial imaging device resolution and huge volume data field. Seeking to speed up the rendering speed, improve the rendering quality, have become the focus in the $3 \mathrm{D}$ visualization technology [4].

Different from the traditional ray casting algorithm, this paper uses a segmentation algorithm based on multiple morphological structure elements to process ICT slice images original data field in the image preprocessing stage. The new algorithm can solve the problems of powerful computing capacity and slow rendering velocity.

\section{ALGORITHM DESCRIPTION}

\subsection{The Ray Casting Algorithm Principle}

The general flow of ray-casting algorithm is: data classification and color assignment -ray tracing and data resampling -image composition. Firstly, a viewing ray casts into the data set from each pixel on the image plane and intersects the volume data set. Secondly, along each ray, a number of sampling points are taken, and the color and opacity at each re-sampling point are interpolated at the eight surrounding points in object space. Finally, the color and opacity of the re-sampling points of each ray are subsequently composited in depth order to obtain the final color value of the pixel $[4,5]$. The schematic diagram is shown in Fig. (1).

The ray casting algorithm can show very rich internal information. But in order to get better rendering effects, it is needed that more light projection data and sampling many times for every light, which have greatly affected rendering speed. Volume rendering method can't change external light and perspective flexibly. Each change means that the whole drawing process needs to start again.

\subsection{A Segmentation Algorithm Based On Multiple Mor- phological Structure Elements}

\subsubsection{Basic Theory of Mathematical Morphology}

Mathematical morphology is developed from set theory and extended to image analysis by Serra. Based on set theory, mathematical morphology is a very important theory, whose operation must be defined by set arithmetic. Its basic idea is to use a certain form of structural elements to measure and extract the corresponding shape of image in order to achieve the right image analysis and recognition purposes. The basic mathematical morphology has four basic morpho- 
logical operators: dilation, erosion, opening and closing operator [6].

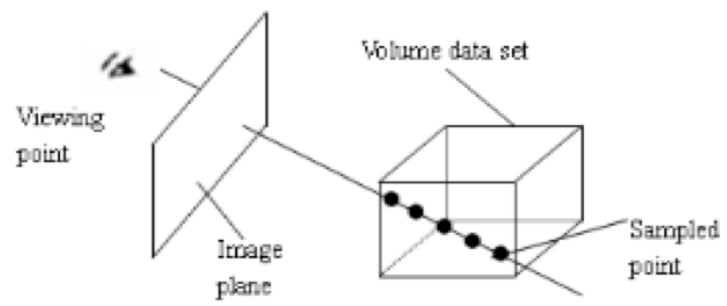

Fig. (1). Sketch map of ray casting algorithm.

The morphologic operations work with two images: The original data to be processed and a structure Element. In the following, we introduce some basic mathematical morphological operators of gray-scale images. Suppose $f(x, y)$ is the function of a gray-scale image in $\mathrm{R}_{2}$ or $\mathrm{Z}_{2}$, suppose $B(x, y)$ is defined structure elements in $\mathrm{R}_{2}$ or $\mathrm{Z}_{2}$ :

The dilation of a gray-scale image $f(x, y)$ by a gray-scale structure element $B(s, t)$ is defined as

$(f \oplus B)(x, y)=\max \{f(x-s, y-t)+B(s, t)\}$

The erosion of a gray-scale image $f(x, y)$ by a gray-scale structure element $B(s, t)$ is defined as

$(f \Theta B)(x, y)=\min \{f(x+s, y+t)-B(s, t)\}$

Opening and closing of a gray-scale image $f(x, y)$ by a gray-scale structure element $B(s, t)$ are defined respectively as

$$
\begin{aligned}
& f \circ B=(f \Theta B) \oplus B \\
& f \bullet B=(f \oplus B) \Theta B
\end{aligned}
$$

Dilation is a transformation of expanding, which filters the outer image, while erosion is a transformation of shrinking, which filters the inner image. But both of them are sensitive to the image edge whose gray-scale value changes obviously. Moreover, the opening suppresses the sharp capes and cuts the narrow isthmuses of the image object, whereas the closing fills up its thin gulfs and small holes. Therefore, morphological operation is used to detect image edge and denoise the image at the same time [7].

\subsubsection{Traditional Morphological Edge Detection}

Morphological edge detection operators can be obtained by combining the four basic operations according to some rules $[8,9]$. Supposes $\mathrm{E}$ as the edge of image, the formula is expressed as:

$$
F_{d}=f \oplus B-f
$$

This is known as dilation residue edge detector, detecting the outside boundary edge. Similarly, the interior boundary edge detector is denoted by:

$F_{e}=f-f \Theta B$

This is also known as erosion residue edge detector.
The morphological gradient of image $f(x, y)$, which denoted by $G(x, y)$, can also be defined as the ifference set of the dilation and erosion, and denoted by

$G(x, y)=(f \oplus B)-(f \Theta B)$

The morphological gradient highlights sharp gray-level transition in the input image, and therefore, it is often used as traditional morphological edge detector. It is simple and easy to implement, but there are also disadvantages. Due to the single structure element and the poor antinoise capability, the edge detected is not complete. We proposed an method as following, to improve noise suppression capability, and detect the edge completely.

\subsubsection{Multiple Structure Elements Morphological Edge Detection}

Modify the above traditional mathematical morphology edge detection operator, as follow.

$$
\begin{aligned}
& F_{\text {max }}(x, y)=\max \left\{F_{d}(x, y), F_{e}(x, y), G(x, y)\right\} \\
& F_{\text {min }}(x, y)=\min \left\{F_{d}(x, y), F_{e}(x, y), G(x, y)\right\} \\
& F_{b}(x, y)=F_{\text {max }}(x, y)-F_{\text {min }}(x, y)
\end{aligned}
$$

The improved mathematical morphology edge detection operator is denoted by :

$F(x, y)=F_{e}(x, y)+0.5 F_{b}(x, y)$

The improved operator can effectively restrain noise and preserve image edge information as superposition of some edge details. In addition, we need to optimize and choose multiple structure elements to filter noise and keep image details simultaneously. The developed algorithm is detecting the image edges with different direction structure elements, and each structure element detects an image edge. So, the exact image edge with detail character can be obtained by combining different orientations, ideal edge can be obtained by choosing different structure elements.

The choosing of structure element is a key factor. The size and shape of structure elements decide the final detected result: the smaller structure element has weak ability to filter noise, but can detect the edge details; the larger structure element has preferable ability to filter noise, but the edge detected is thicker than expected. Generally speaking, the $3 \times 3,5 \times 5$ and $7 \times 7$ window are usually used, among of which $3 \times 3$ window is the fastest, and whose edge is the most exquisite $[10,11]$.

In this paper, we choose:

$$
\begin{aligned}
& B_{1}=\left[\begin{array}{lll}
0 & 1 & 0 \\
1 & 1 & 1 \\
0 & 1 & 0
\end{array}\right], B_{2}=\left[\begin{array}{lll}
1 & 0 & 1 \\
0 & 1 & 0 \\
1 & 0 & 1
\end{array}\right], \\
& B_{3}=\left[\begin{array}{lll}
0 & 0 & 0 \\
1 & 1 & 1 \\
0 & 0 & 0
\end{array}\right], B_{4}=\left[\begin{array}{lll}
0 & 0 & 1 \\
0 & 1 & 0 \\
1 & 0 & 0
\end{array}\right],
\end{aligned}
$$




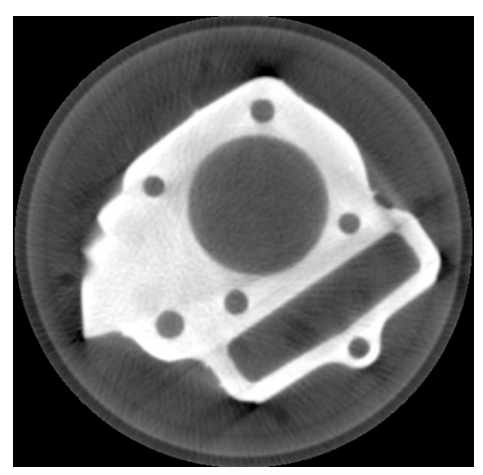

(a) Original ICT image

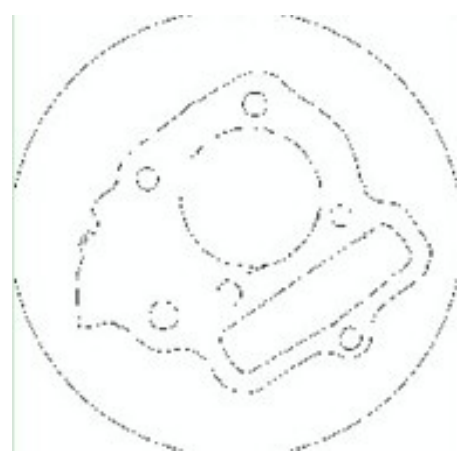

(b) Sobel detection

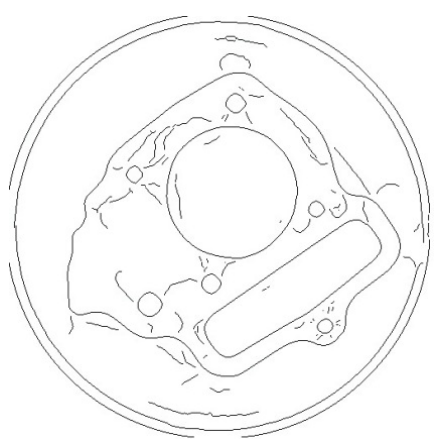

(c) Canny detection

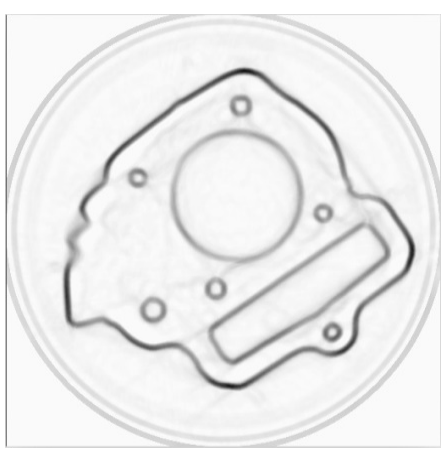

(d) Traditional method detection

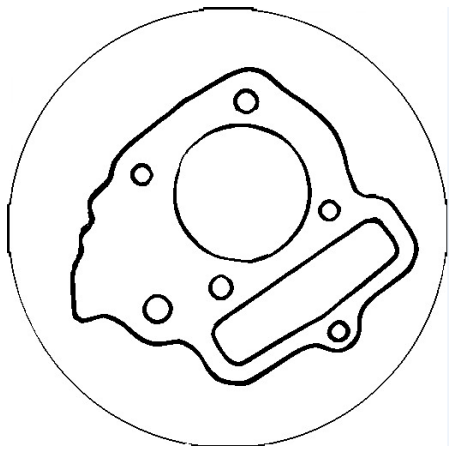

(f) improved method detection

Fig. (2). Edge detection results.

$$
B_{5}=\left[\begin{array}{lll}
0 & 1 & 0 \\
0 & 1 & 0 \\
0 & 1 & 0
\end{array}\right], B_{6}=\left[\begin{array}{lll}
1 & 0 & 0 \\
0 & 1 & 0 \\
0 & 0 & 1
\end{array}\right]
$$

where $B$ is $3 \times 3$ square window, $B_{1}$ is the adjacent pixel template, $B_{2}$ is the weak pixels template, and the direction angles of $B_{3}-B_{6}$ elements are $0^{\circ}, 45^{\circ}, 90^{\circ}$ and $135^{\circ}$. These structure elements comprise almost all the directions of lines extend in the image. By using morphological gradient edge detector respectively, we will get 6 different edge detection results, and the final edge result is got by image fusion using weighted average method.

The improved multiple structure elements morphological edge detection algorithm is compared with several existing edge detection methods by Fig. (2).

The result indicates that the traditional edge detection operator can not availably detect the image edge while the image contain noises, so that the edge is fuzzy and include a great deal of noise. Fig. (2a) is the original ICT image. Figs. (2b-d) are the results of Sobel edge detection, Canny edge detection and the traditional morphological edge detection respectively. Sobel detector can't detect the edge integrally and continually. Though Canny operator detects the edge more continually as shown, it can't differentiate the edge from noise. The traditional morphological edge detector can detect the edge details better than them, but can't filter the noise or detect integral edge. As shown in Fig. (2e), the edge detected by the improved morphological edge detection is more pinpointed, integral and continual than others, and the edge information is more abundant. Moreover, the improved algorithm can filer the noise more successfully than other operators mentioned above.

\subsection{The Improved Ray Casting Algorithm}

In the ray casting algorithm, the more the number of sampling points, the better image quality, but too many sampling points will greatly affect the rendering speed. Generally, the data which can reflect the structure characteristics and composition of the volume dataset are just one part of it. If the data are screened out before rendering and only the voxels which contribute to the rendered image are processed, the three-dimensional images can be rendered quickly and accurately.

To reduce the number of voxels in reconstruction process, the segmentation algorithm based on multiple structure elements is introduced to volume rendering to process ICT slice images original data field. The original voxels are divided into two parts: the target voxel data set and the background voxel data set. Then, the target voxel data set is constructed as a new data field, and using the ray casting algorithm to it. So, the reconstruction volume data can be reduced and the efficiency of ray casting has greatly improved. The flow chart is shown in Fig. (3).

\section{EXPERIMENTAL RESULTS AND ANALYSIS}

The development platform of this paper is Visual Studio 2008 and VTK tools. Using the $\mathrm{C}++$ language program for core algorithm and VTK tools for visualization.

According to the above steps to improve the ray casting algorithm, experiment to $512 * 512 * 512$ motorcycle engine 
industry CT data, the results as shown in Fig. (4). The rendering time of the engine images is reduced to $28693 \mathrm{~ms}$.

\section{ANALYSIS OF BENDING}

In this paper, a segmentation algorithm based on multiple structure elements is proposed to process ICT slice images original data field. Only meaningful target voxels and nodes 


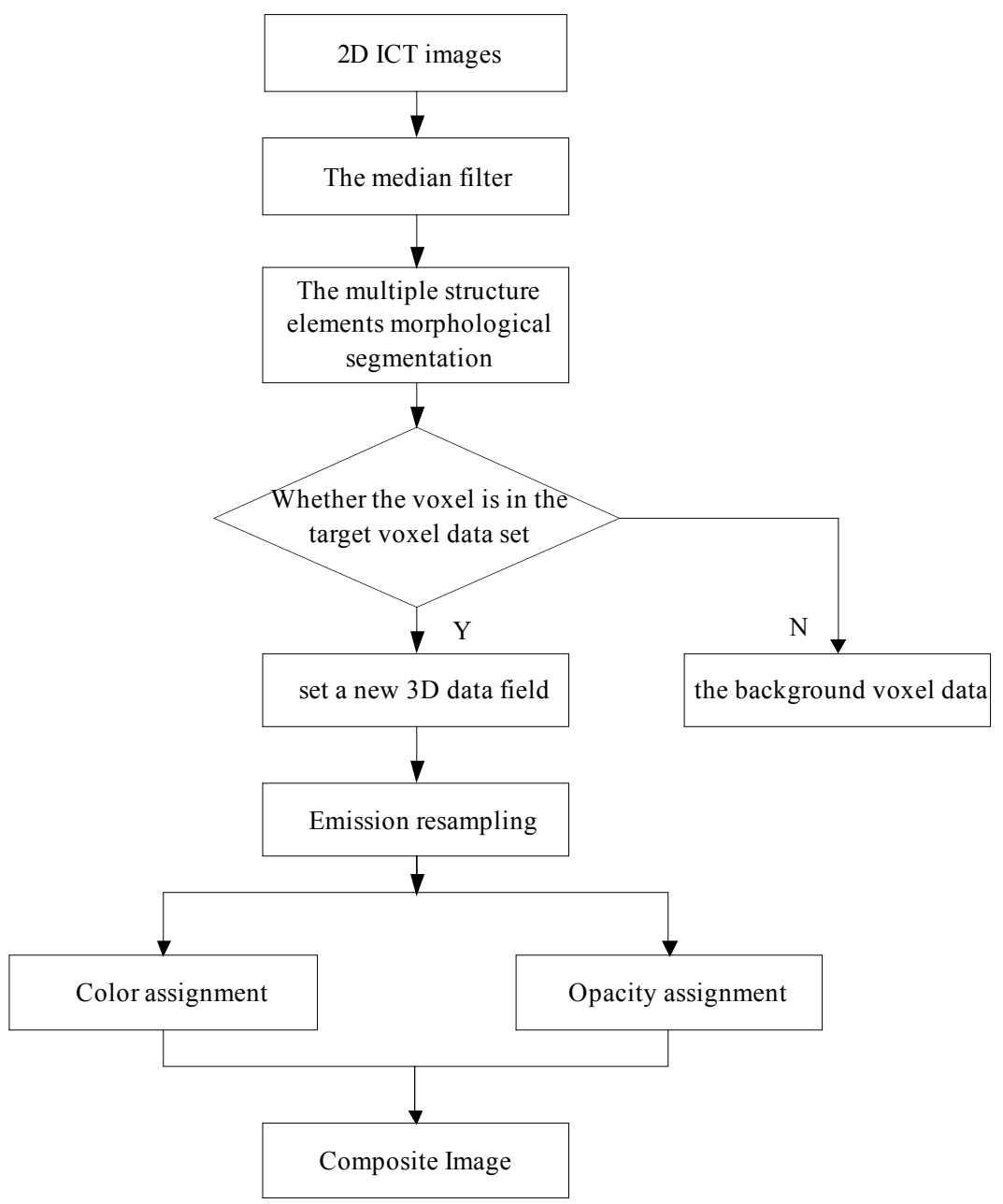

Fig. (3). Improved ray casting algorithm flow chart.

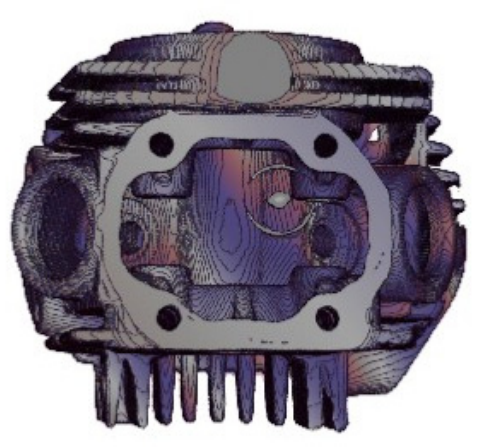

(a) Front display

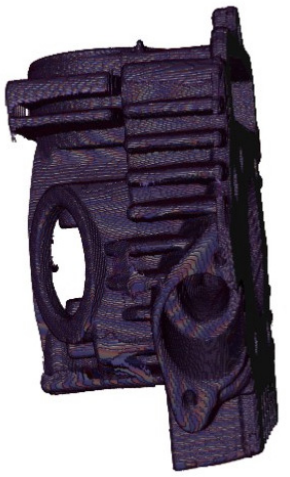

(b) Rolled side display

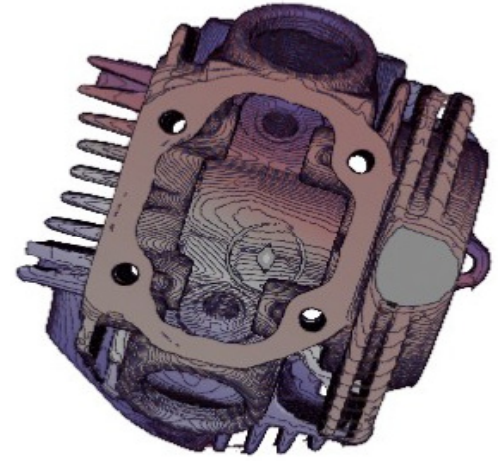

(c) Rolled display

Fig. (4). Experimental results.

for 3D reconstruction are processed and rendered to speed up the rendering speed. The results show that the improved algorithm spend less time, and the effect of the 3D display is satisfactory.

\section{CONFLICT OF INTEREST}

The authors confirm that this article content has no conflict of interest.

\section{ACKNOWLEDGEMENTS}

This work is supported by National Nature Science Foundation of China (No. 61171177).

\section{REFERENCES}

[1] S.T. Ze, "3-D Data Field Visualization", Tsinghua University press, China, 1999

[2] B.H. McCormick, M.D. Brown, "Visualization in scientific", Computer Computer Graphics, vol. 21, no. 6, pp. 1-14, 2007. 
[3] O.P. Marcel, K. Kubin, and T. Mang, "Three-dimensional volume rendering of multi-detector row CT data: applicable for emergency radiology", European Journal of Radiology, vol. 48, no. 1, pp. 33$38,2003$.

[4] G.M. Dai, and Q.H. Li, "Study on some problems of 3-D visualization", Computer Engineering, vol. 28, no. 7, pp. 48-49, 2002.

[5] N. Freud, P. Duvauchelle, and J.M. Letang, "Fast and robust ray casting algorithms for virtual X-ray imaging", Nuclear Instruments and Methods in Physics Research Section B, vol. 248, no. 1, pp. 175-180, 2006.

[6] J. Serra, Image Analysis and Mathematical Morphology, Academic Press: New York, 1982.

[7] H. Yang, and J.W. Zhang "Research on application of mathematical morphology in edge detection of image", Journal of Liaoning University, vol. 32, no. 1, p. 50, 2005.
[8] E. Nalin, and F. Wang, "A morphology algorithm of edge-detection based on multi-structural", Elements, Sclence \& Technology Information, vol. 11, no. 73, 2013.

[9] X. Luo, T. Liu, C. Peng, and W. Li, "An improved morphological edge detection algorithm of medical image based on multi-structure elemen", Journal of Biomedical Engineering, vol. 26, no. 1, pp. 177-180.

[10] C. Niu, H. Fan, and H. Du, "Accelerated algorithm of ray casting in medical volume rendering", Journal of System Simulation, vol. 18, no. 1, pp. 343-346, 2006.

[11] B. Chanda, M. K. Kundu and Y. V. Padmaja, "A mult1-scale morphologic edge detector", Pattern Recognition, Great Britain, vol. 31, pp. 1469-1478, 1998.

Received: September 22, 2014

Revised: November 30, 2014

Accepted: December 02, 2014

(C) Hou et al.; Licensee Bentham Open.

This is an open access article licensed under the terms of the Creative Commons Attribution Non-Commercial License (http://creativecommons.org/licenses/by-nc/3.0/) which permits unrestricted, non-commercial use, distribution and reproduction in any medium, provided the work is properly cited. 